\title{
THE SOLAR WIND INTERACTION WITH VENUS AND MARS: ENERGETIC NEUTRAL ATOM AND X-RAY IMAGING
}

\author{
Mats Holmström ${ }^{1}$ and Esa Kallio ${ }^{2}$ \\ ${ }^{1}$ Swedish Institute of Space Physics (IRF), P.O. Box 812, SE-98128 Kiruna, Sweden \\ ${ }^{2}$ Finnish Meteorological Institute, Geophysical Research, P.O. Box 503, FIN-00101 Helsinki, Finland
}

\begin{abstract}
At the non-magnetized planets Venus and Mars, the solar wind comes in direct contact with the planets' neutrals. This interaction produces energetic neutral atoms (ENAs) and X-rays. In this paper we review some of the different production mechanisms and present examples of simulated ENA and X-ray images. To extract as much information as possible about the ion and neutral distributions in the observed region one has to further process the images. We discuss different approaches to model the production of ENAs and X-rays, and then how to solve the resulting mathematical inverse problems.
\end{abstract}

\section{INTRODUCTION}

Energetic neutral atoms (ENAs) at Mars and Venus are produced from solar wind protons and accelerated planetary ions that charge-exchange with planetary neutrals. Another, less significant, source of ENAs is atmospheric sputtering and back-scattering of precipitating ions and ENAs. In this paper we focus on the ENAs produced from solar wind protons, planetary accelerated oxygen and hydrogen ions that chargeexchange with the hydrogen and oxygen corona.

$\mathrm{X}$-rays are produced by charge-exchange of minor solar wind ions with the exosphere, similar to the Xray production near comets. At Mars and Venus, however, the density of the minor ions does not change significantly during the interaction with the planetary environment, and thus X-rays can be used to visualize the plasma distribution.

Charge-exchange ENA and X-ray images provide global, instantaneous, information about planetary environments. The images depend on the composition and the distribution of the solar wind, planetary plasma and the planetary neutrals. However, it is not straight forward to extract this information from observed images. We need to introduce some prior assumptions about the emissions. One way is to use a model of the production process that depends on a small number of parameters. Given an observed image, it is then possible to extract the parameters that are most likely to have produced the image.

In the case of ENA imaging of planetary ions, since the ENA flux is fully three-dimensional, one has to use non-parametric models, such as tomography. The sputtered, or backscattered, ENAs provide instantaneous maps of the precipitation and are thus directly related to the efficiency of the atmospheric sputtering.

Since Venus and Mars do not have significant global intrinsic magnetic fields, their interaction with the solar wind is similar in many ways. One important difference however, is the presence of crustal magnetizations at Mars that affect the interaction. Also, ENA production at Venus is more intense than at Mars, due to Venus being larger and closer to the Sun, while kinetic effects are less pronounced. That makes Venus particularly attractive for ENA imaging.

Although Venus and Mars recently were imaged using the Chandra X-ray observatory (Dennerl et al., 2002; Dennerl, 2002), detailed imaging of these planets in X-rays and ENAs is an area of future research. 
Table 1. Comparison of the solar wind interaction with Venus and Mars.

\begin{tabular}{l|l|l} 
Property & Difference & Implication \\
\hline Planet radius and volume & 1.8 and 6 times larger at Venus & Larger interaction region \\
Solar wind flux & Four times larger at Venus & Larger production \\
Gravity & Larger at Venus & Different exospheres \\
Internal magnetic fields & Crustal magnetizations at Mars & Modified flow \\
Proton gyroradius & Four times larger at Mars ${ }^{1}$ & Kinetic effects
\end{tabular}

There are two ENA imagers on-board ESA's Mars Express mission, as parts of the ASPERA-3 instrument (Barabash et al., 2002), that will arrive at Mars in December 2003, and similar instrumentation will fly to Venus on-board the ESA Venus Express mission in 2005.

\section{The Solar Wind Interaction with Non-Magnetized Planets}

The solar wind is an outward flow of charged particles, mostly protons and electrons, from the Sun at a speed on the order of $400 \mathrm{~km} / \mathrm{s}$. The density is extremely low by Earth standards, e.g., about 3 protons per $\mathrm{cm}^{3}$ at Mars orbit, but the high flow velocity results in a corresponding flux of $10^{8}$ protons per $\mathrm{cm}^{2}$ each second. The atmosphere of magnetized planets, such as Earth, is shielded from direct interaction with the solar wind by the magnetosphere. For non-magnetized planets, such as Venus and Mars, that lack any significant internal magnetic field, there is no such shielding, and the solar wind comes into direct contact with the upper part of the atmosphere, the exosphere. The resulting collisions between high speed solar wind particles and cold planetary neutrals produce ENAs and X-rays.

Although the interaction with the solar wind is similar for Venus and Mars, there are differences that are important for the planet-solar wind interaction. Some of them, and their implications, are listed in Table 1. We can note that since the crustal magnetizations at Mars are asymmetrically distributed, they will also introduce asymmetries in the solar wind flow around the planet, and in the production of ENAs and X-rays. Also, ion gyroradii at Mars will be about eight times larger compared to the planet radius, than at Venus, making kinetic effects more pronounced at Mars.

\section{X-RAY PRODUCTION MECHANISMS}

There are many possible sources of X-rays from the interaction of the solar wind, and solar X-rays, with atmospheric neutrals near Venus and Mars. Some of the proposed production mechanisms are as follows.

- Fluorescence and scattering of solar X-rays. These two production mechanisms involves solar X-ray photons and atmospheric neutrals. X-rays are produced by fluorescence when photons are absorbed by atmospheric neutrals and then re-emitted isotropically. Solar X-rays can also be elastically scattered by atmospheric neutrals. A detailed, quantitative, analysis of the intensity of the X-rays produced by these two mechanisms has been done by Cravens and Maurellis (2001).

- Solar wind charge-exchange. A small fraction of the solar wind consists of heavy, multiply charged ions such as $\mathrm{O}^{6+}, \mathrm{C}^{6+}$ and $\mathrm{Ne}^{8+}$. Charge-exchange between such an ion and a neutral atom can leave the ion in an excited state. When the captured electron then transits to a lower energy state, within L- and K-shells, X-rays may be emitted. This source of X-rays was first proposed by Cravens (1997) as an explanation of X-rays observed by the Röntgen X-ray Satellite (RosAT) from comet Hyakutake (Lisse et al., 1996), and most likely this process is the dominant source of the soft X-ray emissions from comets (Cravens, 2002). X-rays emitted by this mechanism occur wherever a highly ionized plasma, such as the solar wind, meets a neutral gas, and it was suggested by Cravens (2000) and

\footnotetext{
${ }^{1}$ The factor of four is estimated by comparing IMF magnitude at the planets, taking into account the different Parker spiral angles. We can also note that, for a solar wind proton velocity of $400 \mathrm{~km} / \mathrm{s}$ and an IMF strength of $4 \mathrm{nT}$, the gyroradius at Mars is 0.3 Martian radii.
} 

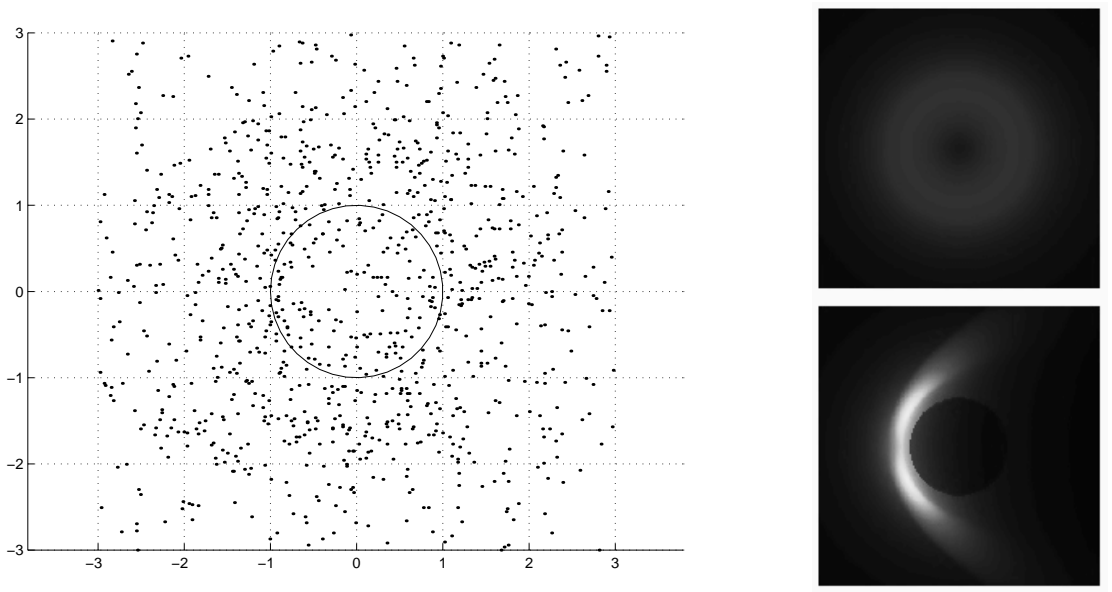

Fig. 1. Computer simulations of X-ray production at Mars from solar wind charge-exchange. To the left, a realization of the spatial distribution of 1000 photons when Mars is at opposition, i.e. photons were randomly drawn with probabilities corresponding to the simulated flux. This is an attempt at visualizing what an X-ray imager would observe from Earth orbit. The scale on the axes are Martian radii. Top right, the corresponding flux. The maximum flux is $5 \cdot 10^{5}\left[\mathrm{eV} /\left(\mathrm{cm}^{2} \mathrm{sr} \mathrm{s}\right)\right]$. Lower right, the flux seen from a direction perpendicular to the Sun-direction, with the Sun to the left. The maximum flux is more than $10^{6}\left[\mathrm{eV} /\left(\mathrm{cm}^{2} \mathrm{sr} \mathrm{s}\right)\right]$. The spectrum of the X-rays is soft, with an average energy of $205 \mathrm{eV}$. Mars is seen as a dark disk in the center. The extent of the images are $6 \times 6$ Martian radii.

Krasnopolsky (2000) that this should be a source of X-ray emissions also at Venus and Mars. Computer simulations of the intensities and morphology of these emissions was presented by Holmström et al. (2001) for Mars, and by Holmström (2001) for Venus. In Figure 1 are shown the results of a simulation of the X-rays produced at Mars by solar wind charge-exchange by the method presented by Holmström et al. (2001).

- Bremsstrahlung and line emissions. Neutrals that are photo-ionized can interact with the solar wind plasma and excite waves, that in turn can accelerate electrons to keV energies. When such electrons collide with neutrals, X-rays can be produced by bremsstrahlung and K-shell radiation. This mechanism has been proposed for cometary X-rays (Bingham et al., 1997; Shapiro et al., 1999), but could also take place at Venus and Mars.

- Dust grain scattering. Dust particles can scatter X-rays efficiently if their size is comparable to the wavelength of the X-rays (Wickramasinghe and Hoyle, 1996). If such particles are present in the atmospheres of Venus or Mars, X-ray emissions could result by scattering of solar X-rays.

\section{ENERGETIC NEUTRAL ATOM PRODUCTION MECHANISMS}

Where energetic ions meet neutral atoms, ENAs are created by charge-exchange. Near the non-magnetized planets the ENA production is large since the solar wind comes into direct contact with the lower, denser, regions of the planets' exospheres. This is in contrast to the situation at Earth where the magnetosphere shields most of the exosphere from the solar wind and only magnetospheric plasma can interact with the neutral gas (Roelof, 1987). Barabash et al. (1995) first estimated the ENA fluxes near Mars. Further studies were made by Kallio et al. (1997).

The charge-exchange process

$$
A_{\text {energetic }}^{+}+M_{\text {cold }} \rightarrow A_{\text {energetic }}+M_{\text {cold }}^{+}
$$

produces ENAs when an energetic (compared to thermal energies) ion, $A^{+}$, collides with a cold (thermal) neutral, $M$, resulting in an ENA and a cold ion. To a good approximation, the produced ENA will have the 

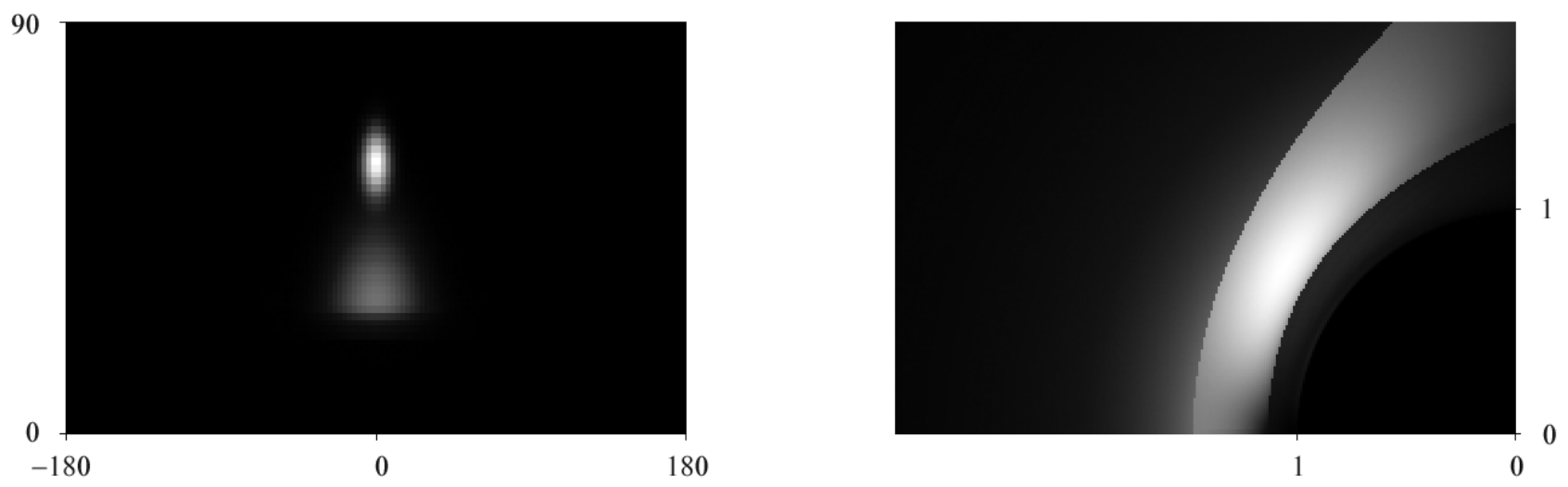

Fig. 2. Computer simulations of hydrogen ENA flux $\left[1 /\left(\mathrm{cm}^{2} \mathrm{sr} s\right)\right]$ at Mars from solar wind protons. To the left, the flux of hydrogen ENAs as seen from a view point at a distance of three Martian radii from the center of Mars, on a line that makes an angle of $120^{\circ}$ to the Mars-Sun line. The maximum flux is $4.6 \cdot 10^{7}$. The image is in a polar format, with azimuth on the x-axis and elevation on the $y$-axis. The look direction is toward the center of Mars. The location of the brightest spot corresponds to the solar direction and is due to ENAs produced in the undisturbed solar wind, upstream of the bow shock. The weaker emissions below corresponds to ENAs produced downstream of the bow shock. The horizontal line at the lower edge of this region is the limb of Mars. To the right, the volume production rate of ENAs in a plane that contain the Mars-Sun axis. The Sun is to the left, and Mars is seen as a dark disk. The visible sunward boundary is the bow shock, while the other boundary is the magnetopause. These emissions produce the lower emission region in the image to the left. The maximum production is $0.02\left[1 /\left(\mathrm{cm}^{3} \mathrm{~s}\right)\right]$. The images are in linear grayscale, so white corresponds to maximum values, and black to minimum.

same velocity as the original ion. Thus, the velocity distribution and volume production of ENAs can be computed if we know the density and velocity distributions of ions and neutrals, and the charge-exchange cross section. Conversely, if we know the neutral density, and the ENA velocity distribution and volume production, we can compute the ion density and velocity distribution. Often the velocity of the produced ENA is large enough so that we can ignore gravity effects. Then the ENAs travel in straight lines since they are unaffected by magnetic and electric fields.

Near Mars and Venus there are several processes that produce ENAs. Solar wind protons interact directly with the planetary neutrals and produce hydrogen ENAs with a velocity distribution similar to that of the parent distribution of protons in the solar wind, i.e. with energies on the order of $1 \mathrm{keV}$ (Holmström et al., 2002). Examples of such simulations are shown in Figure 2. Exospheric neutrals can be ionized by photoionization, electron impact ionization or charge-exchange. The ions are then accelerated by the electric fields, undergo charge-exchange with exospheric neutrals, and produces ENAs, such as hydrogen or oxygen ENAs (Barabash et al., 2002; Lichtenegger et al., 2002). Some ENAs will impact the upper parts of Mars and Venus atmosphere. There, after elastic and inelastic collisions with atmospheric neutrals, some of the precipitated ENAs will be emitted again (backscattered) with a lower velocity (Kallio and Barabash, 2001). This will make the top of the atmosphere glow in ENAs if observed from a distance. Since this effect is quite weak, it is discernible from the solar wind ENAs only when viewing the planet from sunward directions. At Mars, simulations suggest that backscattered ENAs can be seen when the angle between the Sun-Mars line and the view position is smaller than $60 \mathrm{deg}$ (Holmström et al., 2002). At Mars there is an additional source of ENAs. Mars' moon Phobos has a tenuous atmosphere, that produce a gas torus along its orbit around Mars. Charge-exchange between solar wind protons and these neutrals produces hydrogen ENAs that should be observable from favorable positions (Mura et al., 2002). 


\section{IMAGES: OBSERVATIONS}

There have not been many observations of Venus or Mars in X-rays or ENAs. There have been several attempts to image the planets in X-rays, e.g., a ROSAT observation of Mars was attempted in 1993 (ROSAT User's Handbook, 1996), but the result was negative. In hindsight, it seems that the expected count rates during the observation was below ROSAT's sensitivity (Dennerl, 2002) as was predicted by simulations (Holmström, 2001).

The first images of Venus and Mars in X-rays were collected recently, using NASA's Chandra X-ray Observatory (The Chandra Proposers' observatory guide, 2000) that is in Earth orbit (Dennerl et al., 2002; Dennerl, 2002). The observed emissions corresponds well to the sunlit part of the planets' atmospheres in both observations, suggesting that it is fluorescence and scattering of solar X-rays that is responsible for the X-ray emissions. Also, the observed spectra are consistent with that hypothesis (Dennerl et al., 2002; Dennerl, 2002). It is however, interesting to note that for the Mars observation, there are emissions above the background level in an annulus from 1 to 2 Martian radii, that cannot be explained by fluorescence. Also, the same spectrum component of that emission is also weakly visible on the planet disk. A possible source of these emissions is solar wind charge-exchange. An observation of Mars was simulated by Holmström (2001), and the simulated photon distribution is shown in Figure 1. It is consistent with the observations of extended emissions at Mars. Also, the simulated count rates are on the same order of magnitude as the observed ones.

No ENA imaging of Venus or Mars have been done in the past. There has been ENA measurements, e.g., during Cassini's flyby of Venus (Burton et al., 2001), but for higher energies (more than $10 \mathrm{keV}$ for hydrogen) than discussed here. The first ENA images of Mars will be collected by the ASPERA-3 instrument on-board ESA's Mars Express mission that will arrive at Mars in December 2003 (Barabash et al., 2002). ASPERA-3 has two ENA imagers, the neutral particle imager (NPI) with higher angular resolution, but no energy resolution, and the neutral particle detector (NPD) with energy and mass resolution.

Venus will also be imaged in ENAs for the first time, since ASPERA-4, an instrument identical to ASPERA-3, will be part of ESA's Venus Express mission, to be launched in 2005. This will be a unique opportunity for comparative planetology, and will further our knowledge about the similarities, and differences, of the solar wind-planet interaction.

\section{ANALYSIS OF OBSERVED IMAGES}

Imaging gives global measurements. This can be compared with the traditional local measurements (in situ). The advantage of in situ measurements are that they directly give the parameters that usually are of most interest, such as neutral and ion distributions, and field values. On the other hand, the disadvantage is that the measurements are local, so global coverage can only be achieved by using many sensors, or by averaging over time. Also, distinguishing between spatial and temporal features using in situ measurements poses a problem.

The global measurements provided by images, e.g., ENA or X-ray, give instantaneous information about all of the observed region. The drawback here is that what we measure is the integrated flux of ENAs or $\mathrm{X}$-rays along lines-of-sights. If one wants information about the distributions of ions and neutrals, one needs to solve the inverse problem of extracting these distributions from measured images of integrated fluxes.

\section{Image Inversion}

An ENA or X-ray image is essentially a projection of a three-dimensional production function onto the two-dimensional image plane. For now we just count ENAs or photons, if we include energy we have to add another dimension, but the same conclusions follow. To reconstruct this three-dimensional production, without making any further assumptions, is an impossible problem to solve. This is easily seen by an example. What we know about a detected ENA is the direction from where it arrived at the detector. We cannot know where along the line of sight it was produced. Note that the situation would be different if we had multiple images from several well separated view points. Then tomographic methods can be used to reconstruct, at least parts of, the production function.

Thus, we have to introduce further assumptions about the production to be able to solve the reconstruction, or image inversion, problem. This is not unreasonable, since the production function is not arbitrary. We know the physical processes behind the production. One approach is to use a parametric model of the 
production. Such a model for the ENA production near Mars was presented in Holmström et al. (2002). That production model contains 14 parameters for physical quantities such as the solar wind conditions, the flow geometry and the exosphere. The drawback of using a parametric model is that we make the assumption that the physics of the observed system can be captured by the parametric model, thus we cannot observe phenomenas that lies outside the chosen model.

Using a general, non-linear, parametric production model, forward modeling provides a method for extracting parameters from an observed image. That is, given an observed image, we want to find the set of parameter values that produced the image. The idea behind forward modeling is simple: Generate simulated images for different sets of parameters until the simulated image matches the observed image. The generation of simulated images is done by the parametric model (the forward model). The sequence of parameter values is determined by the minimization method used. How do we determine when a simulated image matches an observed image? Ideally, we would also want error estimates on the extracted parameters.

Note that we can use any production model to generate simulated images. There is however the constraint that several hundred (or thousands) of images might need to be generated to analyze one observed image. Thus, to make the problem computationally feasible, the generation of each image must be fast. This precludes the use of more physical correct models, such as MHD models or hybrid models, since it then could take a day, or several days, just to generate one image. Even when given an ion flux, and a neutral distribution, it can take a lot of time to produce an ENA image. One way to speed up this process is to use adaptive methods similar to what is used for volume rendering in computer graphics. Computational experiments suggest that the speed up can be at least an order of magnitude (Laur and Hanrahan, 1991). This is achieved by a spatially adaptive sampling of the production function, with dense sampling only where needed (where the function varies a lot).

\section{Solving the Parametrized Inverse Problem}

In this section we will outline different possible solution methods for obtaining parameter values from an observed image, given that we have a parametrized model that generates simulated images.

We start with $N$ observed data values $n_{i}$. In this case observed pixel counts. From our parametrized model we can generate model values $m_{i}(\beta)$ (simulated pixel counts). These model values depends on $M$ parameters, $\beta_{j}$, i.e. the parameters in our parametrized model.

The question that we want to answer is: Given $n_{i}$, what are likely values of $\beta_{j}$ ? That is, in terms of an observed image: What set of parameters produced this image? The strategy of what is called forward modeling is to find $\beta_{j}$, such that $m_{i}(\beta)$ is close to $n_{i}$ in some sense. For observations $n_{i}$, from a normal distribution, with variance $\sigma_{i}^{2}$, one usually minimizes the chi-square statistic

$$
\min _{\beta} \chi^{2}(\beta), \quad \chi^{2}(\beta)=\sum_{i=1}^{N}\left[\frac{n_{i}-m_{i}(\beta)}{\sigma_{i}}\right]^{2}
$$

The solution is the set of parameters $\beta^{*}$. The advantage of using chi-square minimization is that the functional value at the minimum has a meaning, $\chi^{2}\left(\beta^{*}\right)$ measures the goodness-of-fit between $m_{i}\left(\beta^{*}\right)$ and $n_{i}$, thus it gives information on how well the observed image can be reproduced by the parametrized model. Also, using the chi-square statistics we can compute confidence regions for $\beta_{i}^{*}$, by finding the region in parameter space where $\chi^{2}$ is smaller than some specified value. This approach has been used for analyzing ENA images at Earth (Roelof and Skinner, 2000).

If we have an instrument with energy resolution, we will have to add an integration over energy in the minimization step, or in the discrete case, a summation over several images, corresponding to the different energies. We can also note that the Gaussian chi-square statistic produces the same estimate as a maximum likelihood estimation would produce.

Now, since our observations, $n_{i}$ pixel counts of ENAs or photons, are Poisson distributed, the model counts will be $m_{i}(\beta)=\Delta t \lambda(\beta)$, where $\lambda(\beta)$ is the intensity of the Poisson process (from simulations) and $\Delta t$ is the observation time.

We also need the instrument response function and geometry to compute $m_{i}[\operatorname{counts} / \mathrm{s}]$ from $\lambda\left[\mathrm{eV} /\left(\mathrm{cm}^{2}\right.\right.$ sterad s)]. Many possible $\chi^{2}$-functions can be used (Mighell, 1999), e.g.,

- Pearson's $\chi^{2}$ function, that corresponds to the Gaussian chi-square with $\sigma_{i}^{2}=m_{i}$ since the variance is 
equal to the expectation for a Poisson process,

$$
\chi_{P}^{2}(\beta)=\sum_{i=1}^{N} \frac{\left(n_{i}-m_{i}(\beta)\right)^{2}}{m_{i}(\beta)}
$$

- The chi-square-gamma function,

$$
\chi_{\gamma}^{2}(\beta)=\sum_{i=1}^{N} \frac{\left(n_{i}+\min \left(n_{i}, 1\right)-m_{i}(\beta)\right)^{2}}{n_{i}+1}
$$

An advantage of the chi-square-gamma function is that we avoid the problem of division by zero when $n_{i}$ or $m_{i}$ is zero. Also, it is simpler in the sense that $m_{i}$ only occurs in the nominator.

\section{Choosing Optimization Method}

Minimizing the $\chi_{\gamma}^{2}$-function leads to a weighted least square problem, but the residual is not necessarily small $\left(n_{i}-m_{i}\right.$ can be large). Therefore it is questionable if the standard Gauss-Newton method is applicable, since it linearizes the residuals. Since in the general case $m_{i}(\beta)$ are computed numerically, or by complicated analytical expressions, we cannot assume that analytical gradients are available. Thus, we have to use finite difference gradient approximations (or possibly automated differentiation). Also, the computation of $m_{i}(\beta)$ can be very expensive in terms of computational time. One image has to be generated for each function evaluation, and $M$ images for each gradient approximation. If we have energy resolution, we also have to multiply this by the number of energy levels. This makes a Quasi-Newton optimization method very expensive, since the gradient has to be approximated at each step. Another problem with using local methods is that we are really solving a global optimization problem that possibly has many local minima. An alternative is to use a global method, such as simulated annealing or genetic methods, or start with such a method and then switch to a local method to increase convergence when we are close to a solution.

One method that seems promising is the simultaneous perturbation stochastic approximation (SPSA) method, that only uses two function evaluations at each iteration to obtain an estimate of the gradient. The method has also been shown to be able to solve global optimization problems, and has been applied to magnetospheric ENA image inversion problems (Chin, 1999).

\section{A Non-Parametrized Inverse Problem}

An alternative approach is to try and reduce the dimensionality of the problem. For Mars and Venus, a natural approach would be to assume cylindrical symmetry around the planet-Sun line. If we for ENA images also assume that the ion flux is Maxwellian, we now have a two-dimensional grid where at each grid point the unknowns are the two-dimensional velocity, and temperature of the produced ENAs. This makes the problem solvable, at least in principle, since we now are mapping something two-dimensional (ENA production) onto a two-dimensional image.

This approach can be compared to what has been done when analyzing ENA images at Earth from the IMAGE instrument (DeMajistre et al., 2003). They assume that the ion flux in the equatorial plane determines the ion flux in the whole system, thereby reducing a three-dimensional problem to a two-dimensional, making it solvable.

\section{SUMMARY}

Imaging of ENAs and X-rays at Venus and Mars provides global information about the solar wind-planet interaction, such as ion and neutral distributions. How much information about the planetary environment can be extracted from the images? This question remains to be answered. For ENA images we can hopefully answer the question when ASPERA-3 starts to return images from Mars in 2004, and ASPERA-4 from Venus in 2006. For X-ray images, we will have to wait until missions with X-ray imagers visit these planets.

In this paper, we have presented a general framework to extract information from observed images. There is a lot of freedom in choosing the production model to be used for parameter extraction from images. Before having observed images, it is impossible to predict what method of analysis will be the best. 


\section{REFERENCES}

Barabash, S., R. Lundin, T. Zarnowiecki, and S. Grzedzielski, Diagnostic of energetic neutral particles at Mars by the AsperA-C instrument for the Mars-96 mission, Adv. Space Res., 16, 81-86, 1995.

Barabash, S., M. Holmström, A. Lukyanov and E. Kallio, Energetic neutral atoms at Mars IV: Imaging of planetary oxygen, J. Geophys. Res., 107, 10.1029/2001JA000326, 2002.

Barabash, S., R. Lundin, H. Andersson, et al., ASPERA-3: Analyser of Space Plasmas and Energetic Ions for Mars Express, ESA Special Publication, SP-1240, 2002.

Bingham, R., J. M. Dawson, V. D. Shapiro, D. A. Mendis, and B. J. Kellet, Generation of X-rays from Comet C/Hyakutake 1996 B2, Science, 275, 49-51, 1997.

Burton, M. E., Buratti B., Matson D.Ł., and Lebreton J. P., The Cassini/Huygens Venus and Earth flybys: An overview of operations and results, J. Geophys. Res., 106, 30,099-30,107, 2001.

The Chandra Proposers' Observatory Guide. Version 3.0, Harvard-Smithsonian Center for Astrophysics, Chandra X-ray Center, Cambridge, Massachusetts, 2000,

http://asc.harvard.edu/udocs/docs/POG/MPOG/

Chin, D. C., The Simultaneous Perturbation Method for Processing Magnetospheric Images, Optical Engineering, 38, 606-611, 1999.

Cravens, T. E., Comet Hyakutake X-ray source: Charge transfer of solar wind heavy ions, Geophys. Res. Lett., 24, 105-108, 1997.

Cravens, T. E., X-ray Emission from Comets and Planets, Adv. Space Res., 26, 10, 1443-1451, 2000.

Cravens, T. E., and A. N. Maurellis, X-ray emission from scattering and fluorescence of solar x-rays at Venus and Mars, Geophys. Res. Lett., 28, 15, 3043-3046, 2001.

Cravens, T. E., X-ray Emission from Comets, Science, 296, 1042-1045, 2002.

DeMajistre, R., E. C. Roelof, P. C:son Brandt, and D. G. Mitchell, Retrieval of global magnetospheric ion distributions from high energy neutral atom (ENA) measurements by the IMAGE/HENA instrument, $J$. Geophys. Res., 2003, submitted.

Dennerl, K., V. Burwitz, J. Englhauser, C. Lisse, and S. Wolk, Discovery of X-rays from Venus with Chandra, Astronomy EAAstrophysics, 386, 319-330, 2002.

Dennerl, K., Discovery of X-rays from Mars with Chandra, Astronomy EAAstrophysics, 394, 1119-1128, 2002.

Holmström, M., S. Barabash and E. Kallio, X-ray imaging of the solar wind-Mars interaction, Geophys. Res. Lett., 28, 1287-1290, 2001.

Holmström, M., X-ray imaging of the interaction between the solar wind and non-magnetized planets, Swedish Institute of Space Physics (IRF), Scientific Report 274, March 2001.

Holmström, M., S. Barabash and E. Kallio, Energetic neutral atoms at Mars: I. Imaging of solar wind protons, J. Geophys. Res., 107, 10.1029/2001JA000325, 2002.

Kallio, E., Escaping of Planetary Ions from Mars and Venus, Adv. Space Res., 27, 1815-1824, 2001.

Kallio, E., and S. Barabash, Atmospheric effects of precipitating energetic hydrogen atoms on the Martian atmosphere, J. Geophys. Res., 106, 165-178, 2001.

Krasnopolsky, V., On the deuterium abundance on Mars and some related problems, Icarus, 148, 597-602, 2000.

Laur, D., and P. Hanrahan, Hierarchical splatting: A progressive refinement algorithm for volume rendering, Computer Graphics, 25, 4, 285-288, 1991.

Lichtenegger, H., H. Lammer, and W. Stumptner, Energetic neutral atoms at Mars III: Flux and energy distribution of planetary energetic H atoms, J. Geophys. Res., 107, 10.1029/2001JA000322, 2002.

Lisse, C. M., K. Dennerl, J. Englhauser, M. Harden, F. E. Marshall, M. J. Mumma, R. Petre, J. P. Pye, M. J. Ricketts, J. Schmitt, J. Trümper and R. G. West, Discovery of X-ray and extreme ultraviolet emission from comet C/Hyakutake 1996 B2, Science, 274, 205-209, 1996.

Mighell, K. J., Parameter estimation in astronomy with poisson distributed data. I. The chi-square-gamma statistic, Astroph. J., 518, 380-393, 1999.

Mura, A., A. Milillo, S. Orsini, E. Kallio, and S. Barabash, Energetic neutral atoms at Mars II: Energetic neutral atom production near Phobos, J. Geophys. Res., 107, 10.1029/2001JA000328, 2002.

Roelof, E. C., Energetic neutral atom image of a storm-time ring current, Geophys. Res. Lett., 14, 652-655, 1987. 
Roelof, E. C., A. J. Skinner, Extraction of Ion Distributions from Magnetospheric ENA and EUV Images, Space Sci. Rev., 91, 437-459, 2000.

ROSAT User's Handbook, Max-Planck-Institut für extraterrestrische Physik, Garching, 1996. http://wave.xray.mpe.mpg.de/rosat/doc/ruh/

Shapiro, V. D., R. Bingham, J. M. Dawson, Z. Dobe, B. Kellet, and A. Mendis, Energetic Electrons Produced by the Lower Hybrid Waves in the Cometary Environment and Soft X-ray Emission: Bremsstrahlung and K-Shell Radiation, J. Geophys. Res., 104, 2537-, 1999.

Wickramasinghe, N. C., and Hoyle, F., Very small dust particles (VSDP's) in comet C/1996 B2 (Hyakutake), Astrophys. Space Sci., 239, 121-123, 1996.

E-mail address of M. Holmström is matsh@irf.se 